\title{
Complex wave patterns in an effective reaction-diffusion model for chemical reactions in microemulsions
}

\author{
Sergio Alonso, ${ }^{1, a)}$ Karin John, ${ }^{2}$ and Markus Bär ${ }^{1}$ \\ ${ }^{1}$ Physikalisch-Technische Bundesanstalt, 10587 Berlin, Germany \\ ${ }^{2}$ Laboratoire de Spectrométrie Physique, Université Joseph Fourier, BP 87-38402 Grenoble Cedex, France
}

(Received 1 December 2010; accepted 4 February 2011; published online 7 March 2011)

\begin{abstract}
An effective medium theory is employed to derive a simple qualitative model of a pattern forming chemical reaction in a microemulsion. This spatially heterogeneous system is composed of water nanodroplets randomly distributed in oil. While some steps of the reaction are performed only inside the droplets, the transport through the extended medium occurs by diffusion of intermediate chemical reactants as well as by collisions of the droplets. We start to model the system with heterogeneous reaction-diffusion equations and then derive an equivalent effective spatially homogeneous reactiondiffusion model by using earlier results on homogenization in heterogeneous reaction-diffusion systems [S. Alonso, M. Bär, and R. Kapral, J. Chem. Phys. 134, 214102 (2009)]. We study the linear stability of the spatially homogeneous state in the resulting effective model and obtain a phase diagram of pattern formation, that is qualitatively similar to earlier experimental results for the BelousovZhabotinsky reaction in an aerosol OT (AOT)-water-in-oil microemulsion [V. K. Vanag and I. R. Epstein, Phys. Rev. Lett. 87, 228301 (2001)]. Moreover, we reproduce many patterns that have been observed in experiments with the Belousov-Zhabotinsky reaction in an AOT oil-in-water microemulsion by direct numerical simulations. (C) 2011 American Institute of Physics. [doi:10.1063/1.3559154]
\end{abstract}

\section{INTRODUCTION}

Chemical reaction-diffusion systems are a prominent example of pattern formation outside of equilibrium. ${ }^{1}$ There are many chemical reactions which produce complex spatiotemporal structures. ${ }^{2}$ The Belousov-Zhabotinsky (BZ) reaction was the first system in which target patterns ${ }^{3}$ and rotating spiral waves ${ }^{4}$ were observed. The diversity of patterns observed in chemical systems was increased by the discovery of standing waves and turbulence in the $\mathrm{CO}$ oxidation on a catalytic surface ${ }^{5}$ and the finding of chemical turing patterns in the chlorite-iodide-malonic acid (CIMA) reaction. ${ }^{6}$

However, none of those examples has shown a variety of patterns as large as the one observed in the BZ system dispersed in a water-in-oil aerosol OT (AOT) microemulsion (BZ-AOT system). ${ }^{7-11}$ This heterogeneous system is a dispersion of water-in-oil droplets covered by a surfactant. The small size of the droplets (around several nanometers) ensures that inside the droplets the chemicals are quickly mixed. $^{7}$ While several chemical components of the reaction remain inside the droplets, others components can leave the droplets, diffuse freely in the oil phase, and enter into other droplets.

The most important feature of the AOT system is the possibility to control the diffusion properties of the reacting species by tuning the composition (fractions of water, oil, and surfactant) of the microemulsion. Introducing the composition of the microemulsion as an additional control parameter

\footnotetext{
a) Author to whom correspondence should be addressed. Electronic address:
} sergio.alonso@ptb.de. significantly increases the variety of patterns observed in experiments compared to previous work with the BZ reaction under homogeneous conditions. Upon an increase of the fraction of the disperse phase (water plus surfactant), the system displays an intriguing variety of structures: e.g., static turing patterns, traveling waves, and accelerated waves. ${ }^{7}$ The emergence of turing patterns, in particular, requires largely different diffusion constants for reacting components of the reaction which is achieved by the tuning of the composition of the BZ-AOT system. If, in addition, the chemical composition is also varied, a large variety of complex patterns is obtained experimentally (e.g., inward rotating spiral waves, ${ }^{8}$ stationary and oscillatory localized patterns, ${ }^{10}$ and segmented spirals and target patterns ${ }^{11}$ ).

In the BZ reaction, bromate-oxidate-malonic acid in acidic aqueous solution in the presence of a catalyst. Most of the reaction constituents are polar and, therefore, remain inside the dispersed phase when the reaction occurs in microemulsions. Essentially, the reaction takes place inside the water droplets. There are, however, intermediate species in the reaction which are nonpolar and can diffuse into the oil phase. The most relevant nonpolar intermediates are $\mathrm{Br}_{2}$ and $\mathrm{BrO}_{2}$, which, respectively, inhibits and activates the reaction.

Several models have been proposed to explain the patterns in the BZ-AOT systems. ${ }^{9-11}$ They are typically based on two coupled activator-inhibitor equations, which model the $\mathrm{BZ}$ reaction in the dispersed phase (=water + surfactant), and reaction-diffusion equations with large diffusion coefficients for the nonpolar intermediates which are free to diffuse in the whole system. Numerical simulations with different models reproduced packet waves, ${ }^{9}$ localized patterns, ${ }^{10}$ and dash waves. ${ }^{11}$ However, the models were not systematically 
derived from a given set of heterogeneous conditions and the effect of the fraction of water was so far only phenomenologically included with a linear dependence on the fraction of the homogenized diffusion coefficients. ${ }^{7}$

For a low fraction of the dispersed phase, there is a random distribution of droplets. As this fraction increases, the droplets accumulate and close to the percolation threshold they form clusters. The size of the clusters depends on the fraction of the dispersed phase and arrives to the size of the system for a critical value of the fraction, i.e., the percolation threshold. Percolation in microemulsions can be measured by a sharp increase of the conductivity. ${ }^{12}$

Homogenization techniques constitute a useful approach to study the pattern formation in heterogeneous systems. ${ }^{13-15}$ They allow to derive effective transport coefficients (here diffusion constants) as well as effective reaction rates provided that spatial heterogeneities appear on a scale much smaller than relevant length scales of the emerging reaction diffusion patterns. Earlier work ${ }^{14}$ has shown that the width of a reaction-diffusion front is the relevant scale that decides if homogenization can be applied. Here we apply these techniques to a simple qualitative model inspired by the BZ-AOT system to derive an effective model. The results obtained with this effective model are analyzed systematically and compared with the experimental patterns observed in the BZ-AOT system. We find a good qualitative agreement between simulation results and experimental patterns. We also reproduce the location of the different types of patterns in the parameter space.

The manuscript is organized as follows. In Sec. II, we introduce the model employed in the study. In Sec. III, we calculate the steady states of the model and study its linear stability. Section IV is devoted to show the complex patterns resulting from the numerical simulations. The results are discussed in Sec. V, where they are compared with experimental results existing in the literature. Finally, the main conclusions are stated in Sec. VI.

\section{MODEL}

We consider a three-variable extension of the FitzHughNagumo model for excitable and oscillatory media. A second inhibitor species is added to the standard activator-inhibitor description. Many properties of the homogeneous version of this model have been extensively studied during the last years ${ }^{16-18}$ and similar qualitative models have been already successfully applied to reproduce some of the patterns in the BZ-AOT system. ${ }^{19}$ The equations of the model for a heterogeneous medium read

$$
\begin{gathered}
\partial_{t} u=R(r)\left(a u-\alpha u^{3}-b v-c w\right)+\nabla(D(r) \nabla u), \\
\partial_{t} v=R(r) \epsilon_{1}(u-v)+\nabla(D(r) \nabla v) ; \\
\partial_{t} w=R(r) \epsilon_{2}(u-w)+\nabla\left(D_{w} \nabla w\right) ;
\end{gathered}
$$

where $u$ and $v$ correspond to an activator and inhibitor species restricted to the dispersed phase and $w$ correspond to a species that can diffuse freely in both phases. We consider here that the effect of $w$ is inhibitory, however, for the choice $c<0$ the concentration $w$ activates the reaction. The values of the chemical parameters are constant and homogeneous. The in- homogeneities are introduced through the spatial dependencies of $D(r)$ and $R(r)$. This model formulation qualitatively agrees with the assumptions used to justify the phenomenological models in earlier work. ${ }^{7}$ In contrast to these earlier approaches, we will derive effective equations wherein the parameters depend explicitly on the properties of $R(r)$ and $D(r)$. This will be achieved by employing recently obtained results on homogenization of nonlinear heterogeneous reaction-diffusion systems. ${ }^{14}$

The heterogeneous medium in the above equations is composed of two phases corresponding to water and oil phases of the microemulsion. While the variables $u$ and $v$ are restricted to diffuse and react only inside the water phase, the variable $w$ diffuses in both phases with a homogeneous diffusion coefficient. ${ }^{7}$ To mimic this condition, the function $R(r)$ is assumed to be equal to 1 inside the droplets and 0 outside.

The dispersed phase is formed by water droplets and their surrounding surfactant layers. They are large objects which diffuse in the oil phase with a diffusion coefficient $\left(D_{d}\right)$ much smaller than the diffusion of the chemical species $\left(D_{d} \sim 10^{-7} \mathrm{~cm}^{2} / \mathrm{s}\right.$ and $D \sim 10^{-5} \mathrm{~cm}^{2} / \mathrm{s}$ in experiments $\left.{ }^{7}\right)$. In experiments, this diffusive motion produces contacts between droplets and the interchange of chemical species. We approximate the scenario of moving droplets transporting $u$ and $v$ to an equivalent one where droplets are randomly distributed static heterogeneities. This assumption will be justified in detail below. To take into account the transport by droplet diffusion, we assume that the variables $u$ and $v$ diffuse with a reduced coefficient $D_{d}$ (corresponding to the diffusion of the water droplets) through the oil phase and with large diffusion coefficient $\left(D_{i}\right)$ inside the dispersed phase essentially following the argument given in Ref. 7.

Reactions described in Eq. (1) are assumed to occur only inside the water droplets. To account for this effect, we introduce a parameter $R$ which is $R=R_{i}=1$ inside the droplet and $R=R_{\text {oil }}=0$ outside, giving rise to a heterogeneous reaction term in Eq. (1).

This framework consisting of small static heterogeneities (representing water droplets) randomly distributed in the system, satisfies the conditions to apply a homogenization theory: the characteristic size of the heterogeneities, i.e., droplets (tens of nanometers) is much smaller than the characteristic length of the patterns in experiments, which was found to be always larger than $0.1 \mathrm{~mm} .{ }^{7}$ It ensures that the diffusion time is shorter than the reaction time and, therefore, the chemicals we suppose are completely mixed inside the droplets. ${ }^{14} \mathrm{We}$ assume further that the exchange of material between both phases is faster than the characteristic reaction time.

We apply the homogenization procedure ${ }^{14,15}$ to replace the heterogeneous system by an effective homogeneous system. An effective diffusion coefficient $D_{e}$ for the variables $u$ and $v$ is then obtained from the self-consistent expression,

$$
\frac{D_{d}-D_{e}}{D_{d}+(d-1) D_{e}}(1-\phi)+\frac{D_{i}-D_{e}}{D_{i}+(d-1) D_{e}} \phi=0,
$$

where the homogeneous parameter $D_{e}$ depends on the fraction $\phi$ of the dispersed phase. Expression (2) is not restricted to three-dimensional systems but it can be applied to any spatial dimensionality $d$ of the medium. In Fig. 1(a), the 



(b)

$\phi$

FIG. 1. (a) Dependence on the dispersed phase fraction of the effective diffusion coefficient for a heterogeneous medium composed of two phases with $D_{d}=0.01$ and $D_{i}=2$ for different spatial dimensionality: $d=1$ (black), $d=2$ (red), $d=3$ (green), and $d \approx \infty$ (blue). (b) Dependence on the dispersed phase fraction of the effective reaction rate for a heterogeneous medium composed of two phases with $R_{i}=1$ and $R_{\mathrm{oil}}=0$.

dependence of $D_{e}$ on the water fraction is plotted for four different values of $d$. For the particular case of $d=2$, the explicit expression of the effective diffusion coefficient can be given,

$$
\begin{aligned}
D_{e}(\phi) & =\frac{1}{2}\left(D_{d}-D_{i}\right)(1-2 \phi) \\
& +\frac{1}{2} \sqrt{\left(D_{i}+D_{d}\right)^{2}-4 \phi(1-\phi)\left(D_{i}-D_{d}\right)^{2}} .
\end{aligned}
$$

It was shown in Ref. 15 that Eq. (2) is valid for slowly moving droplets. The dynamics of the droplets is determined by the characteristic time of diffusion of the droplets $\tau_{d}$ $=l^{2} / D_{d}$. If this time is larger than the characteristic time of diffusion of the chemicals inside the droplets $\tau_{i}=l^{2} / D_{i}$, we can apply the expression obtained in Eq. (2). The condition $D_{d}<<D_{i}$ (i.e., $\tau_{d}>>\tau_{i}$ ) justifies the above assumption of static heterogeneities.

The reactions corresponding to local changes of the concentrations $u$ and $v$ take place only inside the droplets. We homogenize the reaction rates ${ }^{14,15}$ to obtain an effective value of $R$ by the averaging of both phases,

$$
R_{e}=R_{i} \phi+R_{\mathrm{oil}}(1-\phi)
$$

and introducing the corresponding values given above, we finally obtain homogeneous reaction rates,

$$
R_{e}(\phi)=\phi
$$

which in contrast with $D_{e}$ is independent of the spatial dimensionality $(d)$ of the medium. The linear dependence of $R_{e}$ on the fraction of dispersed phase is plotted in Fig. 1(b).

Putting together the effective parameters, the equations for the effective model now read

$$
\begin{gathered}
\partial_{t} u=\phi\left(a u-\alpha u^{3}-b v-c w\right)+\nabla\left(D_{e}(\phi) \nabla u\right), \\
\partial_{t} v=\phi \epsilon_{1}(u-v)+\nabla\left(D_{e}(\phi) \nabla v\right) ; \\
\partial_{t} w=\phi \epsilon_{2}(u-w)+\nabla\left(D_{w} \nabla w\right) .
\end{gathered}
$$

It corresponds to a modified FitzHugh-Nagumo model where the dynamics of $u$ and $v$ are controlled by the fraction $\phi$. The inhibitor $w$ diffuses through the whole system. The effect of increasing the fraction is an increase of the reaction rates and diffusion coefficients of variables $u$ and $v$. Thus, by a change of the fraction we control the reaction-diffusion properties of the system.

An important property of the effective model is that it reproduces the percolation transition. If we set $D_{d}=0$, the effective diffusion coefficient in Eq. (2) follows a linear function $D(\phi)=D_{i}(d \phi-1) /(d-1)$ and zero below a critical value of the fraction $\left(\phi_{c}\right)$. For small and finite values of $D_{d}$ two different regions are observed. Above $\phi_{c}$ the effective diffusion coefficient increases approximately linearly with $\phi$, and below $\phi_{c}$ the effective diffusion coefficient remains close to the value $D_{d}$.

The size of the droplets at low fraction of the dispersed phase in the described experiments is clearly smaller than the extension of the system orthogonal to the plane in which patterns are monitored. Therefore, one might be inclined to consider an effective medium theory for a three-dimensional system, which would produce a small percolation threshold $\phi_{c}=0.33$. However, the estimated experimental percolation threshold ( $\left.\phi_{c}=0.4-0.6\right)$ (Ref. 7) is larger and hence is in better agreement with the results from the two-dimensional (2D) approach for the effective medium theory. Following these experimental facts, we employ $d=2$, which produces a reasonable large value of the percolation threshold of $\phi_{c}$ $=0.5$. On the other hand, the percolation threshold depends on the temperature ${ }^{19}$ and close to percolation the dispersed phase cannot be just interpreted as a random distribution of droplets because channels and tubular structures may appear among the droplets. These structural changes would affect the topology of the system. Another reason to study a twodimensional system of model equations here is that most observed patterns appear quasi-two dimensional.

\section{LINEAR STABILITY ANALYSIS}

Equation (6) have three homogeneous steady states. They are given by the solution of the following two conditions:

$$
\begin{gathered}
u_{o}=v_{o}=w_{o}, \\
0=u_{o}\left(a-b-c-\alpha u_{o}^{2}\right) .
\end{gathered}
$$

The trivial state $u_{o 1}=0$ is present always, and the nontrivial steady states, given by

$$
u_{o 2,3}= \pm \sqrt{\frac{a-b-c}{\alpha}} .
$$

It appears if the condition $a>b+c$ is satisfied.

We analyze the linear stability of the solutions of the model. We obtain Hopf, turing, and wave bifurcations, respectively, depending on the parameters. The Jacobian $J_{i}$ of the solution $u_{o i}$ is given by 


$$
\left(\begin{array}{ccc}
\phi\left(a-3 \alpha u_{o i}^{2}\right)-D_{e}(\phi) k^{2} & -\phi b & -\phi c \\
\phi \epsilon_{1} & -\phi \epsilon_{1}-D_{e}(\phi) k^{2} & 0 \\
\phi \epsilon_{2} & 0 & -\phi \epsilon_{2}-D_{w} k^{2}
\end{array}\right) \text {, }
$$

where $k$ denotes the wave number of the perturbation. Note that $D_{e}(\phi)$ depends on the fraction of dispersed phase.

Figure 2 shows the variation of the solution of the homogeneous equations $(k=0)$ with the increase of the autocatalytic parameter $a$ for a constant fraction $\phi$. The solution $u=0$ is the steady state for low values of $a$. For increasing values of $a$, the system crosses a Hopf bifurcation at $a_{H}=2.8$ and oscillates, see Fig. 2(b). The other two solutions $u_{o 2}$ and $u_{o 3}$ appear at $a_{s}=6.5$. Initially they are unstable, see Fig. 2(c), and the system follows a complex limit cycle. These two solutions become stable at $a_{B}=8.35$. They are symmetric and the particular choice of one of them depends on the initial condition, see evolution for two different initial conditions in Fig. 2(d).

A systematic study of the parameter space is done to show the different types of instabilities of the model. We vary the fraction of inhomogeneities $\phi$ and the autocatalytic term $a$ (mimicking experimental phases space ${ }^{20}$ ). The rest of the parameters are kept constant.

We calculate the dispersion relation obtained from the linear stability analysis for each couple of values of $a$ and $\phi$. Some representative examples are plotted in Fig. 3. For a small region of parameters, a wave instability appears, see Fig. 3(a). There is a maximum of the real eigenvalue for $k>0$ with nonzero imaginary eigenvalue. It gives rise to oscillations with a finite wavelength (i.e., waves). The most common



(a)
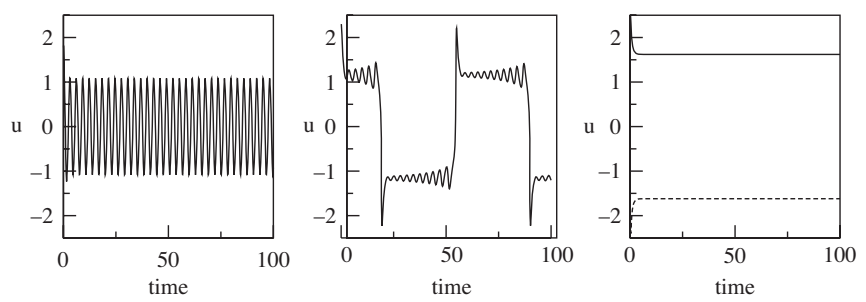

(b)

(c)

(d)

FIG. 2. (a) Solution of Eq. (6) in a zero-dimensional system. Different regimes are observed: Steady state, oscillations (maximum and minimum are shown), and bistability. Three examples of temporal evolution are shown: (b) Oscillations corresponding to $a=4$, (c) irregular oscillations corresponding to $a=8.3$, and (d) bistability corresponding to $a=10$, two different initial conditions are shown. Values of the parameters: $\phi=0.8, b=3, c=3.5$, $\epsilon_{1}=1, \epsilon_{2}=4, \alpha=4 / 3, D_{w}=1, D_{i}=2$, and $D_{d}=0.01$. situation, however, is that the most unstable mode appears at $k=0$, and produces a Hopf instability, see Fig. 3(b). The rest of the panels in Fig. 3 show different examples where a turing instability appears. This instability corresponds to a maximum positive real eigenvalue with a zero imaginary eigenvalue. It gives rise to static spatial patterns (i.e., turing patterns). Several instability can appear at the same time, for example: Hopf/turing in Fig. 3(d), Hopf/wave/turing in Fig. 3(c), and wave/turing in Fig. 3(e).

The complete phase diagram obtained from the linear stability analysis is shown in Fig. 4. For low values of $a$, the system is in the steady state and no pattern is observed. For large values of $a$, the solutions $u_{o 2}$ and $u_{o 3}$ become stable and the model shows bistability. For large values of $\phi$ the system is basically oscillatory and for low values of $\phi$ turing patterns are observed.

The parameters of the model are tuned to obtain wave instability at intermediate values of $\phi$ while at low $\phi$ the turing instability dominates.
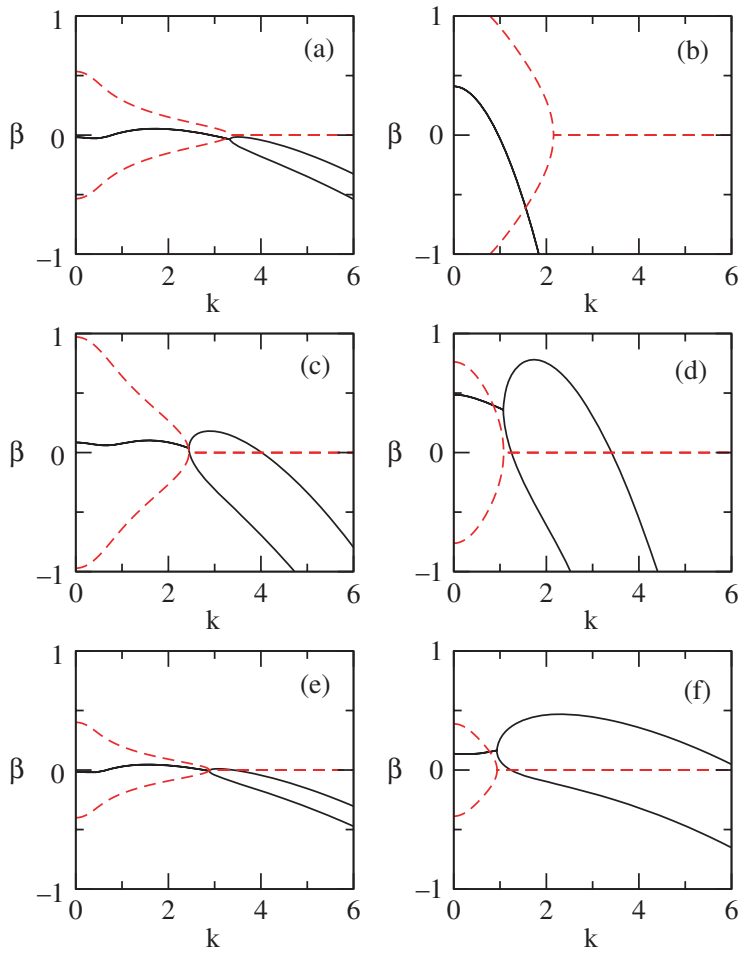

FIG. 3. Dispersion curves obtained by linear stability analysis. Solid and dashed lines correspond to real and imaginary eigenvalues, respectively. The parameter values employed are: $a=2.7$ and $\phi=0.2$ (a), $a=4$ and $\phi=0.6$ (b), $a=3.2$ and $\phi=0.4$ (c), $a=4.5$ and $\phi=0.5$ (d), $a=2.7$ and $\phi=0.15$ (e), and $a=4$ and $\phi=0.2$ (f). Values of the rest of parameters: $b=3$, $c=3.5, \epsilon_{1}=1, \epsilon_{2}=4, \alpha=4 / 3, D_{w}=1, D_{i}=2$, and $D_{d}=0.01$. 


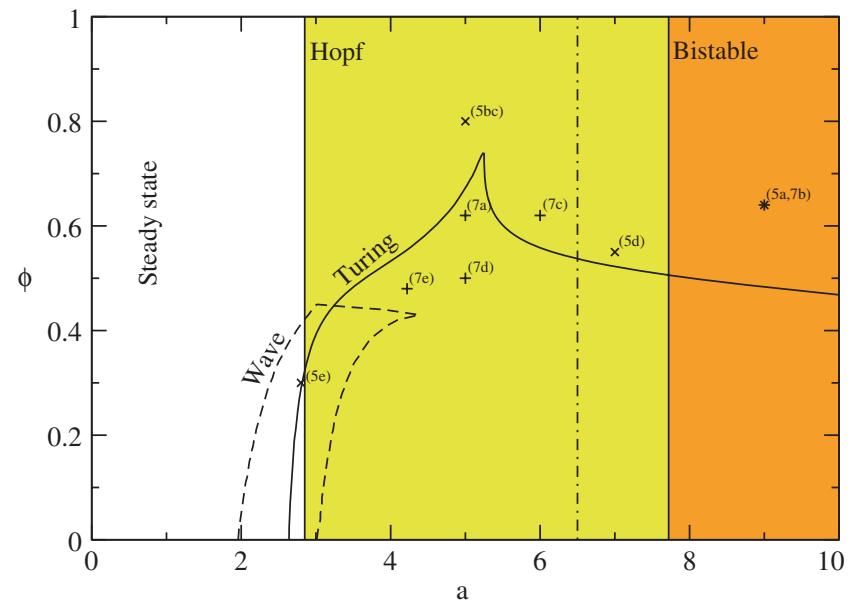

FIG. 4. Phase diagram obtained by linear stability analysis of the solutions obtained in Eqs. (7) and (8). Lines correspond to Hopf (solid), bistability (solid), turing (solid), and wave (dashed) instabilities. Right of the dotteddashed line the two solutions $u_{o 2,3}$ become physically relevant. Crosses $\times$ and + correspond, respectively, to the parameter values employed in the numerical simulations shown in Figs. 5 and 7. Values of the parameters: $b=3$, $c=3.5, \epsilon_{1}=1, \epsilon_{2}=4, \alpha=4 / 3, D_{w}=1, D_{i}=2$, and $D_{d}=0.01$.

\section{NUMERICAL SIMULATIONS}

We integrate Eq. (6) with an explicit method for the temporal integration and finite differences for the Laplacian. The numerical integration of Eq. (6) produces typical dynamics observed in active media. Examples of such patterns are shown in Fig. 5. Front propagation, typical for bistable media, is observed in Fig. 5(a), where one of the corners is initially perturbed. Note, however, that in this case both solutions $u_{o 2}$ and $u_{o 3}$ are symmetric and both are equally stable, the front dynamics is determined by curvature effects. Using the same initial condition but with different values of the parameters transient oscillating cluster are observed in Fig. 5(b), which slowly evolve to global oscillations, see Fig. 5(c). Changing again the parameter values and using a random initial condition static turing patterns in Fig. 5(d) or waves in Fig. 5(e) are obtained.

The spatiotemporal dynamics shown in Fig. 5 is a result from different instabilities of the uniform solution. The homogeneous solution can, however, be unstable to several instabilities at the same time (see Fig. 3) giving rise to more complex dynamics. In the following, we focus on the central area of the phase diagram displayed in Fig. 4. In this region typically several instabilities are present simultaneously. We ran numerical simulations and obtained a gallery of complex patterns, displayed in Fig. 6. We plot the respective state of the system at the end of a numerical simulation for different values of $\phi$ and $a$. The initial conditions are random. Different regions are clearly identified in Fig. 6. For low values of $\phi$ static patterns are observed. For low values of the parameter $a$ they correspond to a conventional turing instability. We observe labyrinthine patterns typical for bistable media ${ }^{21}$ at large values of $a$. Increasing the value of $\phi$ we successively observe a regime of seemingly chaotic patterns stemming from the interactions between unstable turing and Hopf modes. Finally inwardly rotating spiral waves and phase waves are observed (a)
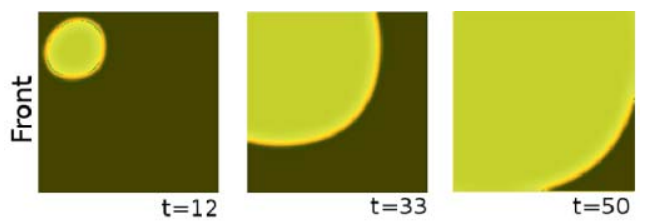

(b)
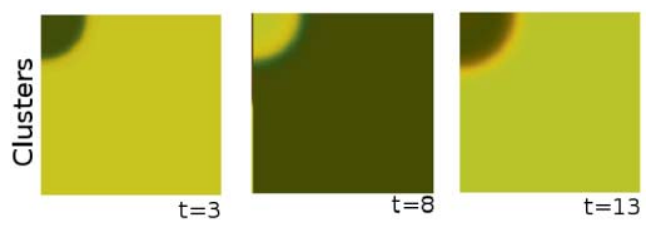

(d)
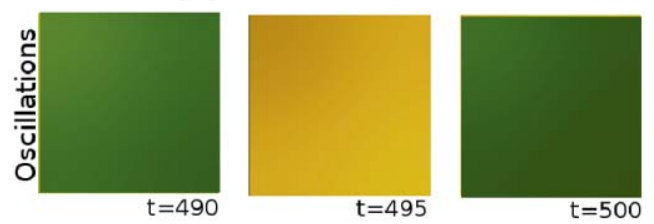

(c)
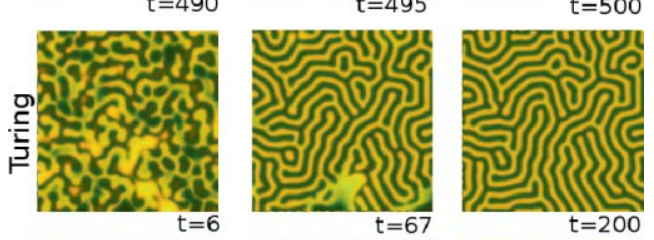

(e)
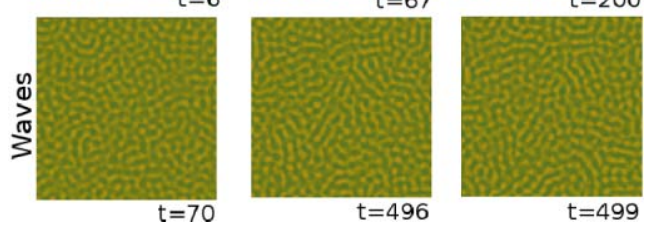

FIG. 5. Typical evolution of different patterns obtained in phase space of Fig. 4 corresponding to: $a=9$ and $\phi=0.64$ (a), $a=5$ and $\phi=0.8$ (b) and (c), $a=7$ and $\phi=0.55(\mathrm{~d})$, and $a=2.8$ and $\phi=0.3$ (e). Bright and dark colors in the snapshots correspond, respectively, to high and low concentrations of the activator. Values of the parameters as in Fig. 4. Numerical parameters: $\Delta x=0.3$ and $\Delta t=0.01$ in a grid of $200 \times 200$ pixels.

at values of $\phi$ close to 1 . For large values of $a$ the increase of $\phi$ produces interactions between temporal and spatial patterns and gives rise to spiral waves, waves moving outward.

Figure 6 shows only the states of the system at the end of the numerical simulations. There are important details during the evolution which are not shown. Patterns appear, grow, and interact among each other. Some examples of the evolution and interaction among patterns are shown in Fig. 7. For example, from disorganized oscillations inward spiral waves (i.e., antispirals) appear and become stable, see Fig. 7(a). Spiral waves are obtained from random initial conditions after a transient front dynamics, see Fig. 7(b). Under some conditions both types of spirals are unstable and give rise to spiral breakup, with a continuous generation and annihilation of new spirals. An example for antispiral waves is shown in Fig. 7(c). For smaller values of $\phi$ localized patches of turing structures appear and travel among disorganized oscillations and pieces of antispiral waves in a very complex way, see example for chaotic waves in Fig. 7(d). They can be initiated directly from the initial condition as in Fig. 7(d) or, depending on the parameter values they can develop from a residual oscillation appearing in a transient turing pattern as shown in Fig. 7(e), where a wave of chaos propagates into a turing pattern. 


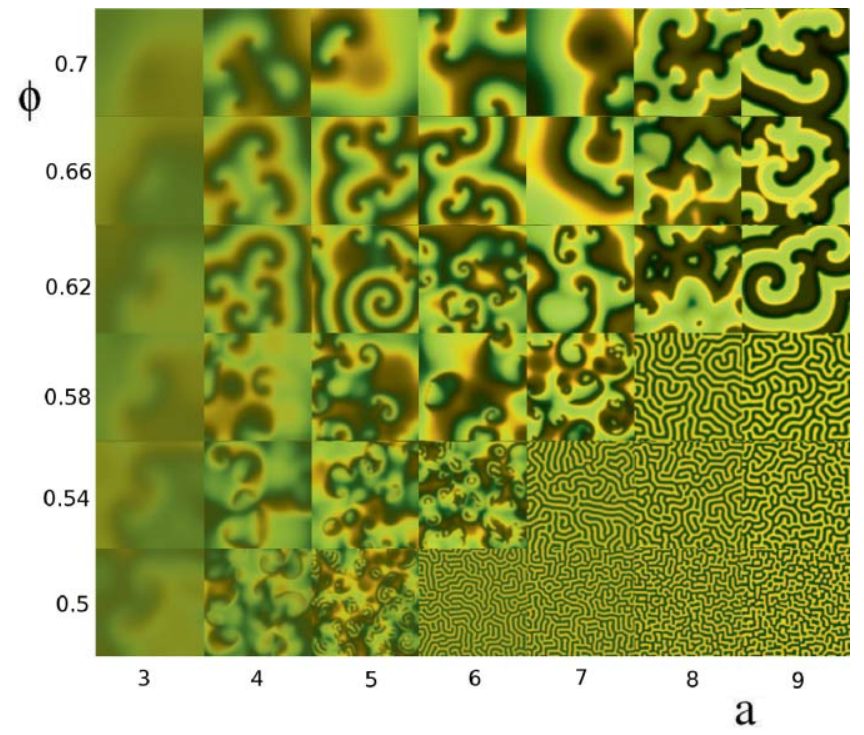

FIG. 6. Collection of patterns obtained at the end of the numerical simulations and arranged as a numerical phase diagram. Bright and dark colors in the snapshots correspond, respectively, to high and low concentrations of the activator. Values of the parameters as in Fig. 4. Numerical parameters: $\Delta x=0.3$ and $\Delta t=0.01$ in a grid of $200 \times 200$ pixels

\section{DISCUSSION}

The reduction from an initially heterogeneous model to an effective homogeneous model was achieved in two steps. First, we have transformed the qualitative model of a chemical medium (i.e., an extended three-variable FitzHugh-Nagumo system) with dynamic heterogeneities (diffusing droplets) to a model with static heterogeneities. Thereby the transport of chemicals through the medium mediated by the motion of the individual droplets below the percolation threshold is reduced to an effective diffusive motion of the respective chemicals. The resulting effective medium theory also reproduces the expected strong increase of the effective diffusion constant above the percolation threshold for the water phase. Second, we have employed a homogenization theory to average the reaction properties inside and outside of the heterogeneities. Some of the employed approximations have already been implicitly assumed in previous models of microemulsions. ${ }^{7,9,11}$

The homogenization procedure resulted in the selfconsistent expressions of Eqs. (2) and (4), which have been previously verified for simple heterogeneous reactiondiffusion systems. ${ }^{14,15}$ Similar expressions have been employed for the calculation of effective properties for the conductivity of isotropic material mixtures ${ }^{22}$ or spherical inclusions in conducting materials. ${ }^{23}$ They have also been employed for inhomogeneous transport problems in resistor networks ${ }^{24}$ and for randomly heterogeneous biological materials. $^{25,26}$

The properties of the patterns formed in heterogeneous media depend on the spatial dimension. This arises from the fact that the effective diffusion coefficients depend on the spatial dimension, whereas the effective (simple) reaction rates do not. Therefore, a change in the spatial dimension changes the aspect of the phase diagram shown in Fig. 4. For the analytical and numerical analyses of the effective model, we (a)
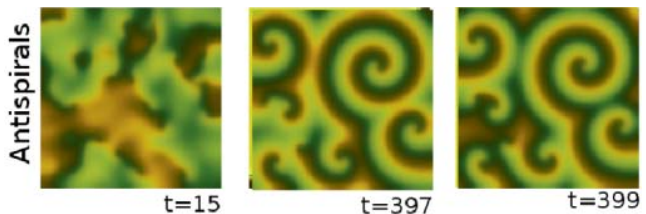

(b)
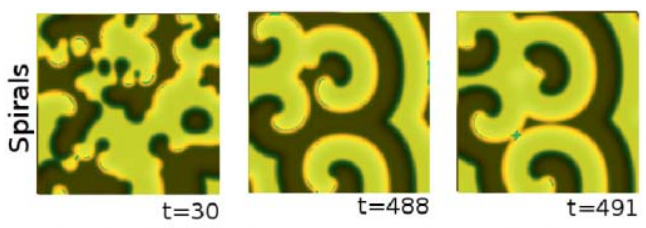

(c)
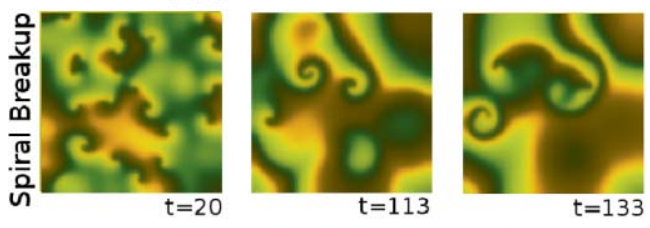

(d)
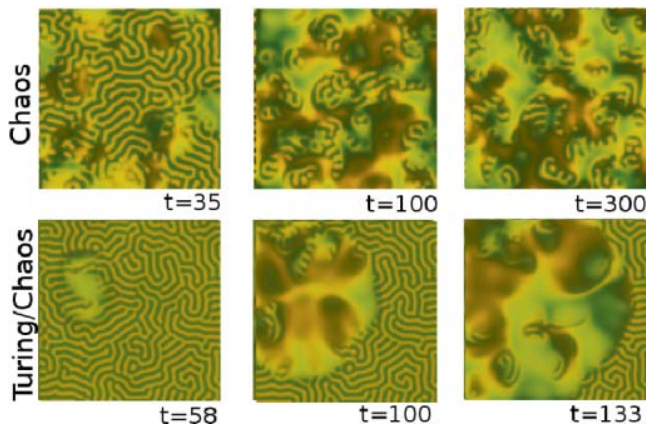

(e)

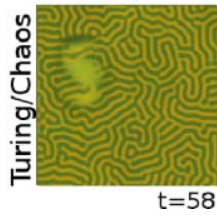

FIG. 7. Evolution of different patterns obtained in phase space of Fig. 4 corresponding to: $a=5$ and $\phi=0.62$ (a), $a=9$ and $\phi=0.64$ (b), $a=6$ and $\phi=0.62$ (c), $a=5$ and $\phi=0.5$ (d), and $a=4.22$ and $\phi=0.48$ (e). Bright and dark colors in the snapshots correspond, respectively, to high and low concentrations of the activator. Values of the parameters as in Fig. 4. Numerical parameters: $\Delta x=0.3$ and $\Delta t=0.01$ in a grid of $200 \times 200$ pixels.

have focused on the 2D case with a fraction around $\phi=0.5$, since the most interesting experiments on the BZ-AOT system were performed in a quasi 2D geometry for similar volume fraction. ${ }^{7-11,20}$

We have studied the dynamical behavior of the effective homogenized system (6) analytically and numerically. The obtained phase diagram spanned by the autocatalytic rate constant and the dispersed phase fraction reproduced qualitatively the experimental phase diagrams of the BZ-AOT system, ${ }^{7,20}$ notably with the occurrence of homogeneous oscillations, oscillating clusters, moving fronts, waves, and turing patterns. However, there are still some discrepancies in the properties of the observed patterns and we do not obtain accelerating waves, ${ }^{7}$ dash waves ${ }^{11}$, and segmented spiral waves. ${ }^{8}$ These discrepancies are a consequence of the simplicity of the simple FitzHugh-Nagumo model employed here. The quality of the predictions of a homogenized model would most probably increase with the use of more detailed chemical modes, e.g., Oregonator models ${ }^{7,27}$ or more elaborate models. ${ }^{28}$

In the interpretation of the experimental studies on the BZ-AOT reaction, it was proposed that the distribution of water droplets might not be completely independent (as we assume in the homogenization) but droplets might aggregate or be connected by channels until percolation occurs, effectively increasing the diffusion of some reactants. Since the 
homogenization process of the transport properties is sensitive to a change in size and nature of the heterogeneities, this phenomenon may change Eq. (2) and alter the phase diagram especially for higher fractions of dispersed phase. However, the modeling of such effects is beyond the scope of the present work.

The theoretical homogenization formalism employed here has been previously checked for simple bistable and excitable reactions using heterogeneous numerical simulations. ${ }^{14,29}$ It is conceivable to consider explicit numerical simulations using heterogeneous reaction-diffusion equations to compare with predictions of the presented effective model. Vice versa the homogenization technique and the related arguments above could be applied to other heterogeneous model with more realistic chemical kinetics, e.g., the heterogeneous BZ (Ref. 30) and CIMA reactions ${ }^{31}$ and different biological systems. . $^{26,32,33}$

The size of the heterogeneities is a relevant factor for the application of the homogenization methodology. In this sense, the nanodroplets formed in the microemulsions provide a straight argument for the validity of a homogenized effective model. Larger sizes of the heterogeneities were achieved in other systems using lithographic techniques, ${ }^{34}$ patterned illumination, ${ }^{35,36}$ reaction droplets in oil,${ }^{37-39}$ or catalystloaded beads. ${ }^{30,40-42}$ The characteristic pattern size in these systems is similar to the size of the heterogeneities and the theoretical approach presented here cannot be applied anymore. In the latter case, however, it is possible to employ smaller beads. ${ }^{43}$ Under such conditions, the methods used here might be extended to the study of some of the experimental results.

\section{CONCLUSIONS}

The application of homogenization techniques derived in Refs. 14 and 15 to a simple model of the BZ-AOT system, permits the reproduction of many of the characteristic patterns observed in the experiments. The organization of the different experimental patterns in the parameter space determined by the autocatalytic rate and the fraction of dispersed phase, is qualitatively reproduced by the phase diagram of this simple model. For a more quantitative study of the patterns, a more complex model could be considered.

In summary, the application of the homogenization procedure to a simple BZ-AOT reaction model qualitatively explains the emergence of a variety of pattern formation in microemulsions and reproduces the experimental dependence of the pattern type on the fraction of dispersed phase and on the chemistry of the system.

\section{ACKNOWLEDGMENTS}

Stimulating discussions with R. Kapral are gratefully acknowledged. We acknowledge financial support from the German Science Foundation Deutsche Forschungsgemeinschaft (DFG) within the framework of SFB 555 "Complex Non- linear Processes" and SFB 910 "Control of Self-Organizing Nonlinear Systems."

${ }^{1}$ Chemical Waves and Patterns, edited by R. Kapral and K. Showalter (Kluwer Academic, Dordrecht, 1993).

${ }^{2}$ F. Sagués and I. R. Epstein, Dalton Trans. 7, 1201 (2003).

${ }^{3}$ A. N. Zaikin and A. M. Zhabotinsky, Nature (London) 225, 535 (1970).

${ }^{4}$ A. T. Winfree, Science 175, 634 (1972).

${ }^{5}$ S. Jakubith, H. H. Rotermund, W. Engel, A. von Oertzen, and G. Ertl, Phys. Rev. Lett. 65, 3013 (1990).

${ }^{6}$ V. Castets, E. Dulos, J. Boissonade, and P. De Kepper, Phys. Rev. Lett. 64, 2953 (1990).

${ }^{7}$ V. K. Vanag and I. R. Epstein, Phys. Rev. Lett. 87, 228301 (2001).

${ }^{8}$ V. K. Vanag and I. R. Epstein, Science 294, 835 (2001).

${ }^{9}$ V. K. Vanag and I. R. Epstein, Phys. Rev. Lett. 88, 088303 (2002).

${ }^{10}$ V. K. Vanag and I. R. Epstein, Phys. Rev. Lett. 92, 128301 (2004).

${ }^{11}$ V. K. Vanag and I. R. Epstein, Proc. Natl. Acad. Sci. U.S.A. 100, 14635 (2003).

${ }^{12}$ M. S. Baptista and C. D. Tran, J. Phys. Chem. B 101, 4209 (1997).

${ }^{13}$ D. Bedeaux and R. Kapral, J. Chem. Phys. 79, 1783 (1983).

${ }^{14}$ S. Alonso, R. Kapral, and M. Bär, Phys. Rev. Lett. 102, 238302 (2009).

${ }^{15}$ S. Alonso, M. Bär, and R. Kapral, J. Chem. Phys. 131, 214102 (2009).

${ }^{16}$ C. P. Schenk, M. Or-Guil, M. Bode, and H.-G. Purwins, Phys. Rev. Lett. 78, 3781 (1997).

${ }^{17}$ E. M. Nicola, M. Or-Guil, W. Wolf, and M. Bär, Phys. Rev. E 65, 055101(R) (2002).

${ }^{18}$ T. Teramoto, X. Yuan, M. Bär, and Y. Nishiura, Phys. Rev. E 79, 046205 (2009).

${ }^{19}$ A. A. Cherkashin, V. K. Vanag, and I. R. Epstein, J. Chem. Phys 128, 204508 (2008).

${ }^{20}$ I. R. Epstein and V. K. Vanag, Chaos 15, 047510 (2005).

${ }^{21}$ A. Hagberg, A. Yochelis, H. Yizhaq, C. Elphick, L. Pismen, and E. Meron, Physica D 217, 186 (2006).

${ }^{22}$ D. A. G. Bruggeman, Ann. Phys. 416, 636 (1935).

${ }^{23}$ S. Torquato, Random Heterogeneous Materials (Springer, New York, 2002).

${ }^{24}$ S. Kirkpatrick, Rev. Mod. Phys. 45, 574 (1973).

${ }^{25}$ O. P. Posnansky and N. J. Shah, J. Biol. Phys. 34, 551 (2008).

${ }^{26} \mathrm{~S}$. Alonso, M. Bär, and A. V. Panfilov, "Effects of reduced discrete coupling on filament tension in excitable media," Chaos (in press).

${ }^{27}$ V. K. Vanag and I. R. Epstein, J. Chem. Phys. 131, 104512 (2009).

${ }^{28}$ A. M. Zhabotinsky, F. Buchholtz, A. B. Kiyatkin, and I. R. Epstein, J. Phys. Chem. 97, 7578 (1993).

${ }^{29}$ S. Alonso, J. Löber, M. Bär, and H. Engel, Eur. Phys. J. Spec. Top. 187, 31 (2010).

${ }^{30}$ R. Toth and A. F. Taylor, J. Chem. Phys. 125, 224708 (2006).

${ }^{31}$ X. Yuan, X. Lu, H. Wang, and Q. Ouyang, Phys. Rev. E 80, 066201 (2009).

${ }^{32}$ K. H. W. J. Ten Tusscher and A. V. Panfilov, Europace 9, vi38 (2007).

${ }^{33}$ D. E. Postnov, F. Müller, R. B. Schuppner, and L. Schimansky-Geier, Phys. Rev. E 80, 031921 (2009).

${ }^{34}$ O. Steinbock, P. Kettunen, and K. Showalter, Science 269, 1857 (1995).

${ }^{35}$ I. Sendiña-Nadal, S. Alonso, V. Pérez-Muñuzuri, M. Gómez-Gesteira, V. Pérez-Villar, L. Ramírez-Piscina, J. Casademunt, J. M. Sancho, and F. Sagués, Phys. Rev. Lett. 84, 2734 (2000).

${ }^{36}$ H. Skodt and P. G. Sorensen, Phys. Rev. E 68, 020902(R) (2003).

${ }^{37}$ M. Toiya, V. K. Vanag, and I. R. Epstein, Angew. Chem. Int. Ed. 47, 7753 (2008).

${ }^{38}$ M. Toiya, H. O. Gonzalez-Ochoa, V. K. Vanag, S. Fraden, and I. R. Epstein, J. Phys. Chem. Lett. 1, 1241 (2010).

${ }^{39}$ S. Thutupalli, S. Herminghaus, and R. Seemann, Soft Matter 7, 1312 (2011).

${ }^{40}$ A. F. Taylor, M. R. Tinsley, F. Wang, Z. Huang, and K. Showalter, Science 323, 614 (2009).

${ }^{41}$ M. R. Tinsley, A. F. Taylor, Z. Huang, and K. Showalter, Phys. Rev. Lett. 102, 158301 (2009).

${ }^{42}$ G. Wang, Q. Wang, P. He, S. Pullela, M. Marquez, and Z. Cheng, Phys. Rev. E 82, 045201(R) (2010).

${ }^{43}$ J. Maselko, J. S. Reckley, and K. Showalter, J. Phys. Chem. 93, 2114 (1989). 